\title{
Ajustes morfológicos e fisiológicos em plantas jovens de Araucaria angustifolia (Bertol.) Kuntze em resposta ao sombreamento
}

\author{
Ana Maria Soares Franco ${ }^{1}$ e Lúcia Rebello Dillenburg ${ }^{1,2}$
}

Recebido: 18.05.2006; aceito: 15.02.2007

\begin{abstract}
Morphological and physiological adjustments in young plants of Araucaria angustifolia (Bertol.) Kuntze in response to shading). This study evaluated the ability of plants of Araucaria angustifolia in adjusting some aspects of their morphology and physiology to different light conditions. Plants were submitted to full irradiance and shaded $(90 \%$ reduction) conditions. During the course of a greenhouse experiment, four harvests were made (31, 49, 75 and 158 days after planting) and growth measurements of the shoot and roots were taken. A reduction in dry mass accumulation was observed in the shaded plants. These plants also had an initial enhancement of height growth, accumulated more chlorophyll, and, by the end of the experiment, had branched less and attained a greater leaf area ratio than the control plants. These morphological and physiological changes, which exhibited a temporally discontinuous expression, were interpreted as adjustments towards a more efficient light absorption and resource conservation under shading, which may contribute to the reported hade tolerance of the species.
\end{abstract}

Key words: Brazilian pine, light, shade tolerance

RESUMO - (Ajustes morfológicos e fisiológicos em plantas jovens de Araucaria angustifolia (Bertol.) Kuntze em resposta ao sombreamento). Este trabalho avaliou a capacidade de plantas de Araucaria angustifolia em ajustar alguns aspectos de sua morfologia e fisiologia a diferentes condições de luz. As plantas foram submetidas a irradiação plena e sombreamento (redução de 90\%). Ao longo do experimento, conduzido em casa de vegetação, foram efetuadas quatro avaliações $(31,49,75$ e 158 dias após o plantio) de crescimento na parte aérea e no sistema radicular. Uma redução no acúmulo de massa seca foi verificada nas plantas sombreadas. Estas também exibiram maior crescimento inicial em altura, acumularam mais clorofila e, ao final do experimento, haviam ramificado menos e atingido maior razão de área foliar, quando comparadas às controles. Estas alterações morfológicas e fisiológicas, cujas expressões foram temporalmente descontínuas, foram interpretadas como ajustes para uma absorção mais eficiente de luz e de economia de recursos sob condições de sombreamento, devendo contribuir para a relatada tolerância ao sombreamento da espécie.

Palavras-chave: luz, pinheiro brasileiro, tolerância à sombra

\section{Introdução}

Araucaria angustifolia (Bertol.) Kuntze (Araucariaceae), também conhecida como pinheiro brasileiro ou pinheiro-do-paraná, é uma das essências florestais mais importantes no sul do Brasil. A espécie destaca-se pela alta qualidade de sua madeira, fonte de alimento para a fauna e para o homem, como também pela exuberante beleza paisagística na região onde ocorre (Reitz \& Klein 1966).

A exploração irracional e descontrolada de sua valiosa madeira nos últimos 40 anos reduziu a área de ocupação da espécie, de cerca de $200.000 \mathrm{~km}^{2}$ para aproximadamente $6.000 \mathrm{~km}^{2}$. Essências exóticas (em sua maioria dos gêneros Pinus e Eucalyptus) têm sido selecionadas para projetos de reflorestamento, em substituição à araucária, devido à rapidez de crescimento nas condições edafo-climáticas da região sul (Tomaselli 1980). Diante desta situação, torna-se imprescindível promover sua regeneração natural, bem como estimular seu uso em reflorestamentos, visando sua preservação e o uso sustentável de seus recursos econômicos. Para isso, estudos sobre os fatores que afetam a regeneração desta espécie, assim como a caracterização de seus ajustes morfofisiológicos frente às condições ambientais, são de primordial importância

1. Universidade Federal do Rio Grande do Sul (UFRGS), Instituto de Biociências, Departamento de Botânica, Av. Bento Gonçalves 9500, Campus do Vale, 91509-900 Porto Alegre, RS, Brasil

2. Autor para correspondência: lucia.dillenburg@ufrgs.br 
para orientar procedimentos de regeneração artificial.

Um dos fatores ambientais de grande importância para o estabelecimento de uma espécie arbórea é a disponibilidade de luz. A habilidade de algumas espécies em manter populações de plântulas e plantas jovens no sub-bosque de florestas está associada à capacidade das mesmas de incrementar a sobrevivência sob baixas irradiâncias, ou seja, tolerar o sombreamento (Walters \& Reich 2000). Segundo estes autores, esta capacidade de sobrevivência aumenta com o tamanho da semente e pela expressão de caracteres morfológicos e fisiológicos que promovem crescimento sob reduzida disponibilidade luminosa. Dentre estes caracteres, cabe destacar o aumento das taxas fotossintéticas líquidas sob baixas irradiâncias, uma diminuição na razão clorofila a/ clorofila $b$, uma menor razão de massa entre raiz e parte aérea, uma maior área foliar específica, maiores razões de massa e área foliar, dentre outros, que funcionalmente resultariam em melhor aproveitamento da baixa radiação disponível (Boardman 1977, Givnish 1988, Lei \& Lechoiwicz 1998, Poorter 1999). Um aumento na capacidade de crescimento sob baixa intensidade luminosa como um componente da tolerância ao sombreamento é apontado como lógico, uma vez que aumentaria a capacidade competitiva destas plantas (Walters \& Reich, 1999). No entanto, mais recentemente, a idéia de que a capacidade de crescer com pouca luz é uma característica que sempre confere tolerância ao sombreamento tem sido questionada e reformulada, e a capacidade de sobreviver na sombra (que pode ser favorecida pela manutenção de um crescimento líquido global positivo) vem assumindo um papel de maior destaque na caracterização da tolerância ao sombreamento, principalmente de plântulas (Walters \& Reich 1999 , 2000, Lusk 2004).

A araucária demonstrou ser capaz de ocupar, sobreviver e crescer, mesmo em taxas distintas, tanto ambientes ensolarados quanto sombreados, em experimentos conduzidos em casa de vegetação (Backes 2001, Inoue et al. 1979, Inoue \& Torres 1980, Duarte \& Dillenburg 2000) e a campo (Duarte et al. 2002), contrapondo-se à classificação da espécie como pioneira e heliófila, sem capacidade de desenvolvimento e, consequentemente, de regeneração em ambientes sombreados (Reitz \& Klein 1966). Inúmeras características podem contribuir de forma significativa para essa habilidade da espécie, incluindo o tamanho avantajado de suas sementes (pinhões) e a capacidade de ajustar processos fisiológicos e padrões morfológicos em resposta a variações na disponibilidade de luz. Assim, este trabalho visa quantificar alterações em parâmetros tanto morfológicos quanto fisiológicos de conhecida relevância no processo de tolerância ao sombreamento, em plantas jovens de A. angustifolia submetidas a diferentes condições de disponibilidade de luz. Comparado a estudos anteriores (Inoue et al. 1979, Inoue \& Torres 1980, Duarte \& Dillenburg 2000), o presente trabalho procurou ampliar o número de características investigadas e acompanhá-las ao longo do desenvolvimento inicial da espécie.

\section{Material e métodos}

Condições de cultivo - Os pinhões utilizados no experimento foram coletados na Floresta Nacional de São Francisco de Paula (FLONA), em São Francisco de Paula (RS), entre as coordenadas geográficas $29^{\circ} 23^{\prime}$ a $29^{\circ} 27^{\prime} \mathrm{S}$ e $50^{\circ} 23^{\prime}$ a $50^{\circ} 25^{\prime} \mathrm{W}$. Após a coleta, os pinhões foram desinfestados com hipoclorito de sódio 2,5\% durante 30 minutos, enxaguados em água e parcialmente escarificados (remoção dos envoltórios em cerca de $1 / 3$ da porção proximal do pinhão), com o objetivo de tornar a germinação mais rápida e uniforme (Áquila \& Ferreira 1984). Os pinhões foram colocados em bandejas contendo vermiculita autoclavada e úmida. Dez dias após o início da germinação, quando as radículas alcançaram aproximadamente $4,5 \mathrm{~cm}$, os pinhões pré-germinados foram transferidos individualmente para recipiente de cultivo (vaso de PVC com 39,5 cm de altura e $10 \mathrm{~cm}$ de diâmetro), contendo uma mistura de substrato orgânico comercial (Plantmax, Eucatex), areia média (previamente lavada), solo proveniente de mata localizada na Universidade Federal do Rio Grande do Sul (Porto Alegre, RS) e solo de mata com araucária, coletado na FLONA de São Francisco de Paula (RS), na proporção de 4:3:2,5:0,5 (v:v:v:v), respectivamente.

No experimento, foram utilizadas 80 plantas, distribuídas em dois tratamentos: plantas controle (C) e plantas sombreadas (S), onde as plantas de cada tratamento receberam $100 \%$ e $10 \%$ da luz disponível, respectivamente. Para efeito de sombreamento, as plantas S foram mantidas sob duas camadas de Sombrite. Duas medições de irradiância foram realizadas, com um sensor de quanta de luz (LI-190S-1) de um porômetro de estado estacionário (modelo LI-1600, Licor Inc.). A primeira medição foi feita aos 
40 dias após o plantio (setembro/2001), e a segunda aos 149 dias (janeiro/2002). Os valores médios de irradiância (medidos entre 11:00 e 13:00 horas) para as plantas $C$, na primeira e segunda medição, foram de 694,3 e $1277,3 \mu \mathrm{mol} \mathrm{m} \mathrm{m}^{-2} \mathrm{~s}^{-1}$, respectivamente. As plantas S sofreram uma redução de $90 \%$ em relação às plantas $\mathrm{C}$. $\mathrm{O}$ delineamento experimental foi o de blocos inteiramente casualizados, sendo cada planta em um vaso tratada como unidade experimental. De acordo com o comprimento da raiz, medido antes do plantio, as plantas foram inicialmente separadas em dez blocos, todos contendo quatro plantas de cada tratamento. Para a avaliação dos parâmetros morfológicos das plantas, foram realizadas quatro avaliações ao longo do experimento: 31, 49, 75 e 158 dias após o plantio. Para cada avaliação, 20 plantas foram sorteadas, dez de cada tratamento (uma de cada bloco).

Avaliação das Plantas - foram feitas medições semanais de altura (ramo principal) e de comprimento total da parte aérea (ramo principal + laterais). Em cada uma das quatro avaliações, as plantas foram separadas em parte aérea (folhas + caule) e raízes (laterais + principal). Foram, então, realizadas medições de massa seca total da parte aérea; área foliar total, usando um de medidor portátil de área (Modelo LI 3100, LI-COR); massa foliar por área (MFA, razão entre massa seca e área foliares); grau de ramificação (razão entre comprimento total de ramos laterais e altura da parte aérea); e comprimento caulinar específico (CCE, razão entre comprimento total da parte aérea e massa seca caulinar). Para o sistema radicular, foram feitas medidas de comprimento de raiz (principal e laterais); massa seca total de raiz (principal + laterais); e comprimento radicular específico de raiz principal + raízes laterais (CRE, razão entre comprimento e massa seca). Com os dados de parte aérea e raízes, foram analisados os seguintes parâmetros: razão entre massa seca da parte aérea e sistema radicular e razão de área foliar (razão entre área foliar e massa seca total da planta). As medidas de comprimento foram feitas com auxílio de uma régua milimetrada. Para as medidas de massa, as plantas foram secas em estufas a $90{ }^{\circ} \mathrm{C}$ até peso constante e, posteriormente, pesadas em balança analítica (Sartorius, modelo 2842). O conteúdo de clorofila foi quantificado somente na $2^{\mathrm{a}}$ e $3^{\mathrm{a}}$ avaliações, devido a problemas metodológicos nas outras duas avaliações. A clorofila foi extraída de cinco acículas frescas de cada planta (preferencialmente as medianas do eixo caulinar) em etanol absoluto
(Knudson et al. 1977), e as leituras de absorbância foram efetuadas a 649 (clorofila $b$ ) e $665 \mathrm{~nm}$ (clorofila a), em espectrofotômetro (PM 2K, Zeiss). A concentração de clorofila foi calculada segundo Wintermans (1965).

Análise Estatística - Os dados foram analisados no programa estatístico Sigmastat (SPSS Institute Inc., version 2.03), através de Análise de Variância (Anova), usando-se como fontes de variação bloco e tratamento e adotando-se um nível de 5\% de significância para o valor de $F$.

\section{Resultados e Discussão}

De acordo com o esperado, o sombreamento, ao final do experimento, induziu menor acúmulo de massa seca total, tanto na parte aérea quanto no sistema radicular (tabela 1). A ausência de diferenças no acúmulo de massa nas avaliações anteriores à final, em particular nas duas primeiras, deve estar diretamente associada ao vínculo ainda funcional da plântula ao pinhão, vínculo este que tipicamente perdura cerca de 3 a 4 meses (observação pessoal). Interessantemente, verificou-se, nos dois grupos de plantas, uma redução na massa radicular, particularmente entre a segunda e terceira avaliações. Esta redução foi revertida nas plantas controle, mas não nas plantas sombreadas. Nos dois tratamentos, esta redução esteve associada a uma diminuição na massa da raiz principal (dados não mostrados). Sugere-se que a raiz pivotante da espécie esteja atuando no estoque de reservas oriundas do pinhão, com posterior suprimento destas reservas para o crescimento da parte aérea e das raízes laterais. Em Araucaria cunninghammi, foi caracterizado um padrão pouco usual de germinação criptógea (Burrows 1992), onde a emissão da parte aérea só ocorre após a total transferência das reservas do pinhão, via cotilédones, para o hipocótilo subterrâneo, o qual se transforma em um tubérculo, de onde, então, emerge a parte aérea. Embora este não seja o padrão de germinação da $A$. angustifolia, é possível que seu hipocótilo subterrâneo (cuja massa é tipicamente computada na massa radicular, devido à sua continuidade e semelhança física em relação à porção basal da raiz principal) esteja atuando como um importante dreno inicial das reservas do pinhão. Estudos futuros deverão abordar este aspecto.

As menores massas acumuladas na parte aérea das plantas $\mathrm{S}$ ao final do experimento resultaram tanto 
Tabela 1. Parâmetros diversos de plantas de A. angustifolia em resposta aos tratamentos de luz, nas diferentes avaliações. Médias seguidas de letras diferentes são significativamente diferentes $(\mathrm{P} \leq 0,05)$. MFA: massa foliar por área; $\mathrm{Cl}$ : clorofila; CRE: comprimento radicular específico; CCE: comprimento caulinar específico, CRT: comprimento radicular total.

\begin{tabular}{|c|c|c|c|}
\hline Parâmetro & Dias após plantio & Plantas controle & Plantas sombreadas \\
\hline \multirow[t]{4}{*}{ Massa seca parte aérea $(\mathrm{g})$} & 31 & 0,22 a & 0,26 a \\
\hline & 49 & 0,49 a & 0,48 a \\
\hline & 75 & 1,09 a & 0,78 a \\
\hline & 158 & 2,65 a & $1,32 \mathrm{~b}$ \\
\hline \multirow[t]{4}{*}{ Massa seca raiz (g) } & 31 & 0,52 a & 0,55 a \\
\hline & 49 & 0,54 a & 0,46 a \\
\hline & 75 & 0,46 a & $0,34 \mathrm{a}$ \\
\hline & 158 & 0,69 a & $0,31 \quad b$ \\
\hline \multirow[t]{4}{*}{ Raiz/parte aérea ( $\left.\mathrm{g} \mathrm{g}^{-1}\right)$} & 31 & $2,37 \mathrm{a}$ & $2,18 \mathrm{a}$ \\
\hline & 49 & 1,21 a & 0,99 a \\
\hline & 75 & 0,47 a & 0,35 a \\
\hline & 158 & 0,29 a & $0,25 \mathrm{a}$ \\
\hline \multirow[t]{4}{*}{$\operatorname{CCE}\left(\mathrm{cm} \mathrm{g}^{-1}\right)$} & 31 & 34,43 a & 33,85 a \\
\hline & 49 & 58,41 a & 58,17 a \\
\hline & 75 & 58,97 a & 82,46 a \\
\hline & 158 & 50,19 a & 87,06 b \\
\hline \multirow[t]{4}{*}{ Número de folhas } & 31 & 36,10 a & 37,46 a \\
\hline & 49 & $60,20 \mathrm{a}$ & 62,62 a \\
\hline & 75 & $121,22 \mathrm{a}$ & 108,22 a \\
\hline & 158 & 310,8 a & $191,27 \mathrm{~b}$ \\
\hline \multirow{4}{*}{ Área foliar total $\left(\mathrm{cm}^{2}\right)$} & 31 & 7,64 a & 8,90 a \\
\hline & 49 & 24,79 a & $26,83 \mathrm{a}$ \\
\hline & 75 & 63,71 a & 59,17 a \\
\hline & 158 & 163,69 a & $101,81 \mathrm{~b}$ \\
\hline \multirow[t]{4}{*}{$\operatorname{MFA}\left(\mathrm{g} \mathrm{m}^{-2}\right)$} & 31 & 85,69 a & $76,04 \mathrm{a}$ \\
\hline & 49 & 85,19 a & $72,19 \mathrm{~b}$ \\
\hline & 75 & 95,71 a & 81,47 a \\
\hline & 158 & 94,40 a & 82,61 a \\
\hline \multirow[t]{4}{*}{ Razão área foliar $\left(\mathrm{cm}^{2} \mathrm{~g}^{-1}\right)$} & 31 & 10,29 a & $10,83 \mathrm{a}$ \\
\hline & 49 & 22,56 a & 26,43 a \\
\hline & 75 & 44,21 a & 49,13 a \\
\hline & 158 & 48,39 a & $63,18 \mathrm{~b}$ \\
\hline \multirow[t]{2}{*}{ Cl. total $\left(\mathrm{mg} \mathrm{g}^{-1}\right)$} & 49 & 5,34 a & 8,41 b \\
\hline & 75 & 4,75 a & $9,24 \mathrm{~b}$ \\
\hline \multirow[t]{2}{*}{ Cl. a/b } & 49 & 1,41 a & $1,24 \mathrm{a}$ \\
\hline & 75 & 1,62 a & 1,44 a \\
\hline \multirow[t]{4}{*}{ CRT (cm) } & 31 & 74,75 a & $102,89 \mathrm{a}$ \\
\hline & 49 & $130,41 \mathrm{a}$ & 122,19 a \\
\hline & 75 & $154,95 \mathrm{a}$ & $125,08 \mathrm{a}$ \\
\hline & 158 & $192,69 \mathrm{a}$ & $122,90 \mathrm{~b}$ \\
\hline \multirow[t]{4}{*}{$\operatorname{CRE}\left(\mathrm{cm} \mathrm{g}^{-1}\right)$} & 31 & $144,69 \mathrm{a}$ & $186,93 \mathrm{a}$ \\
\hline & 49 & 259,11 a & $282,37 \mathrm{a}$ \\
\hline & 75 & 359,06 a & $364,54 \mathrm{a}$ \\
\hline & 158 & 276,32 a & $395,35 \mathrm{~b}$ \\
\hline
\end{tabular}

de uma redução na massa caulinar quanto na foliar (dados não mostrados). Estas reduções em massa vieram acompanhadas de uma forte tendência $(\mathrm{P} \leq 0,06)$ de um menor comprimento total da parte aérea (figura 1B) e de uma menor área foliar total das plantas (tabela 1), sendo que esta menor área foliar foi causada por uma redução no número de folhas e não na área individual das mesmas (dados não mostrados). Além da esperada redução na massa e tamanho das plantas S, o padrão de crescimento destas plantas também diferiu daquele apresentado pelas plantas C. Até aproximadamente 110 dias após o 
plantio, as plantas $\mathrm{S}$ cresceram significativamente mais em altura (figura 1A). Após este período, mesmo apresentando maior CCE (tabela 1), evidenciando um maior grau de estiolamento caulinar, as plantas $\mathrm{S}$ estabilizaram o crescimento em altura, de forma que, nas últimas avaliações, este parâmetro não mais diferia entre os dois grupos de plantas. Ao contrário, as plantas $\mathrm{C}$ mantiveram um crescimento contínuo em altura e apresentaram um maior investimento em ramos laterais (figura $1 \mathrm{C}$ ).

Um maior investimento no crescimento em altura em ambientes sombreados é uma resposta bastante comum, relatada em vários trabalhos (Cancian \& Cordeiro 1998, Poorter 1999, Ferrer \& Dillenburg 2000, Jurado et al. 2006), inclusive para a espécie em estudo (Inoue \& Torres 1980). No entanto, verifica-se que a vantagem de altura das plantas sombreadas sobre as controle é transitória. Espécies que apresentam caules de pequeno diâmetro (evidenciado nas plantas sombreadas, pelos seus maiores valores de CCE), juntamente com pouco investimento na ramificação da parte aérea, são capazes de se alongar mais rapidamente para um determinado regime de irradiância, devido às baixas quantidades de massa requeridas por unidade de crescimento em altura (Takahashi 1996). O investimento inicial pronunciado no alongamento vertical do caule e uma reduzida ramificação da parte aérea são considerados respostas fenotípicas adaptativas (Poorter 1999, Niinemets et al. 2006), apesar de um estiolamento pronunciado (alto CCE) muitas vezes comprometer tanto a sustentação da parte aérea quanto o investimento em ramos laterais, por reduzir o vigor do caule (Steingraeber 1982). Assim, um possível significado ecológico do maior investimento inicial em altura apresentado pela espécie, quando cultivada sob sombra, consistiria em sobrepujar mais rapidamente a vegetação concorrente, o que poderia garantir o seu sucesso regenerativo (Inoue \& Torres 1980). No entanto, Ryser \& Eek (2000) salientam que o aumento no crescimento em altura pode ser vantajoso como resposta a um sombreamento de curto prazo. Este seria, por exemplo, o caso de uma planta emergindo de um dossel herbáceo ou arbustivo ou de uma densa camada de serrapilheira. No caso de um sombreamento contínuo, como o estabelecido no presente estudo e como aquele que se estabelece no sub-bosque florestal, o mais vantajoso, segundo os mesmos autores, seria a conservação dos recursos por parte da planta. Assim, num primeiro momento, um rápido crescimento em altura estaria associado principalmente a plantas intolerantes ao sombreamento, conforme tem sido sugerido na literatura (Morgan \& Smith 1979, Brokaw 1987). Cabe salientar que, mesmo reduzindo
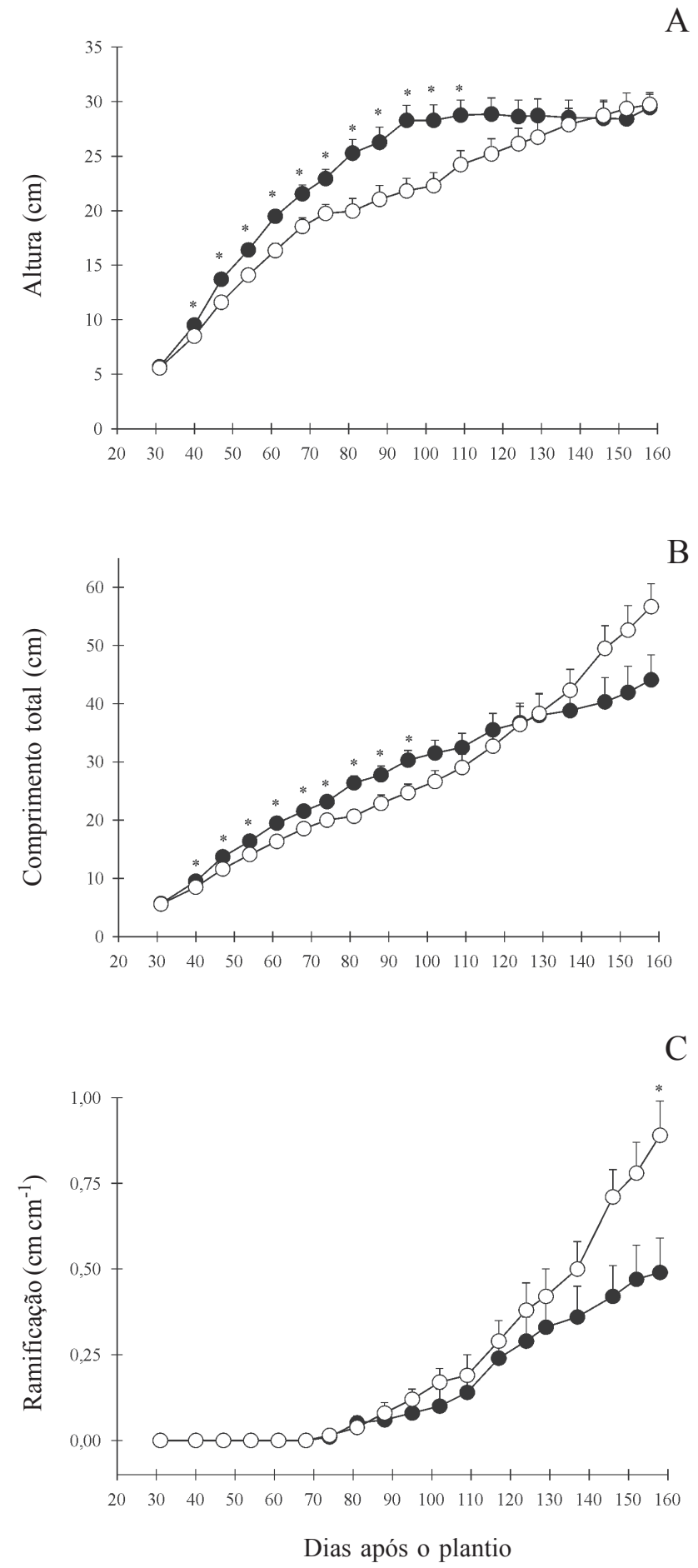

Figura 1. Variações na altura (A), comprimento total (B) e grau de ramificação (C) da parte aérea de plantas de A. angustifolia ao longo do experimento. As barras verticais indicam o erro padrão da média. $\mathrm{O}$ asterisco indica diferença estatística entre os tratamentos $(\mathrm{P} \leq 0,05)$. S: sombra; $\mathrm{C}$ : controle. $\left(-\bullet-=\mathrm{S} ;-\mathrm{O}_{-}=\mathrm{C}\right)$ 
drasticamente o alongamento das estruturas caulinares, as plantas $\mathrm{S}$ mantiveram, até o final do experimento, um CCE significativamente maior que as plantas controle, indicando uma maior eficiência no uso da massa para alongamento dos eixos caulinares (Poorter 1999). Estes são os responsáveis pela extensão tanto vertical quanto horizontal do espaço ocupado pelas folhas. Dependendo das características do perfil vertical de distribuição da luz em uma floresta, um pequeno ganho de altura de uma árvore instalada no sub-bosque pode ser indiferente para o seu ganho de carbono ou levá-la acima do ponto de compensação de luz da fotossíntese. Assim, justifica-se o aumento no comprimento caulinar específico mesmo em plantas tolerantes ao sombreamento (Poorter 1999), as quais podem passar toda ou boa parte de suas vidas sob a sombra do dossel florestal. Assim, tanto o rápido incremento inicial em altura quanto a posterior redução no crescimento global da parte aérea (com manutenção de uma alta eficiência no alongamento das estruturas caulinares), apresentados pela $A$. angustifolia no presente estudo, seriam respostas favoráveis da espécie ao sombreamento.

As plantas expressaram uma redução significativa na MFA em resposta ao sombreamento apenas na segunda avaliação (tabela 1). Em contraste com o presente estudo, Duarte \& Dillenburg (2000) detectaram diferenças significativas entre plantas sob alta e baixa irradiâncias após cerca de 150 dias de cultivo, tempo de cultivo bastante semelhante ao do presente estudo. No entanto, o padrão de amostragem foi diferente nos dois estudos: neste os valores de MFA expressam os valores de todas as folhas presentes no corpo da planta, enquanto que no estudo anterior apenas folhas maduras, recentemente expandidas, foram coletadas. É possível que a inclusão de folhas mais velhas tenha reduzido possíveis diferenças entre folhas maduras, recentemente expandidas. Outra possibilidade é que as diferenças nos resultados ente os dois experimentos estejam associados a efeitos interativos entre irradiância e outros fatores ambientais, como propriedades do solo. Reduções na MFA em resposta à sombra têm sido comumente relatadas (e.g., Callaway 1992, Barret \& Fox 1994, Luken et al. 1995, Wahl et al. 2001). Uma baixa MFA, aliada a outras propriedades das folhas, tem sido historicamente interpretada como uma característica que permite às plantas tolerantes ao sombreamento crescer mais na sombra do que plantas intolerantes, por expressar um menor investimento de massa seca foliar em superfície de absorção de fótons de luz (Boardman 1977, Givnish 1988 Barret \& Fox 1994). No entanto, Lusk (2004) enfatizou o fato de que muitos estudos têm apontado para um baixo potencial de ganho de carbono de plântulas de espécies arbóreas tolerantes à sombra, o que se deve à presença de folhas com elevada MFA, mesmo sob baixas irradiâncias. Segundo o autor, este tipo de comportamento se contrapõe à teoria de Givnish (1988), que propõe que a adaptação à sombra é determinada principalmente por características que aumentam a captura de luz. Assim, mais recentemente, tem sido sugerido que a sobrevivência de plântulas sob baixas irradiâncias é altamente dependente da presença de tecidos duros e resistentes a estresses físicos e pouco atraentes a inimigos naturais (Walters \& Reich 1999, Lusk 2004). Assim, uma fraca resposta da MFA a baixas irradiâncias em $A$. angustifolia pode refletir um mecanismo de sobrevivência baseado na economia de recursos e não na priorização do ganho de carbono.

Em relação aos conteúdos foliares totais de clorofila (tabela 1), estes foram significativamente maiores nas plantas $\mathrm{S}$ do que nas plantas $\mathrm{C}$, nas duas avaliações realizadas. Em apenas uma das duas avaliações (aquela correspondente à segunda avaliação de crescimento) detectou-se diferença significativa na razão ente clorofilas $a$ e $b$ entre os dois tratamentos, sendo esta razão menor nas plantas que cresceram sob sombra. $\mathrm{O}$ aumento na concentração de clorofila por unidade de massa em plantas expostas a baixas irradiâncias é uma resposta consistentemente observada (Boardman 1977, Barrett \& Fox 1994, Nicotra et al. 1997). A razão entre clorofila $a$ e $b$, por sua vez, foi proposta como indicadora do ambiente de luz de uma planta (Dale \& Causton 1992), sendo tipicamente menor em plantas que crescem em condições de sombreamento, considerando que o papel da clorofila $b$ é otimizar o funcionamento no fotossistema II sob baixas irradiâncias (Koike et al. 2001, Nakazono et al. 2001). Neste estudo, as plantas sombreadas de $A$. angustifolia mostraram-se plásticas em relação ao acúmulo e proporções da clorofila, diferindo dos resultados anteriormente obtidos por Duarte \& Dillenburg (2000). No entanto, as avaliações feitas no trabalho anterior estiveram restritas ao período final do experimento, enquanto que as aqui apresentadas correspondem a períodos mais curtos de tratamento. Uma vez que as respostas de MFA foram transitórias, é possível que uma 
avaliação das clorofilas ao final do experimento aqui realizado também tivesse resultado em diferenças insignificantes entre os tratamentos de irradiância.

As menores massas acumuladas no sistema radicular pelas plantas $\mathrm{S}$ ao final do experimento estiveram associadas tanto a um menor comprimento total de raízes (tabela 1), quanto a um maior CRE destas (caracterizando raízes menos espessas e/ou densas que as plantas $\mathrm{C}$ ). A redução no comprimento radicular total deveu-se a uma redução no número e comprimento das raízes laterais (dados não mostrados). Embora a literatura relate um menor número de estudos enfocando os efeitos da disponibilidade de luz sobre a estrutura e funcionamento radicular do que sobre a parte aérea, estes apontam para um maior investimento em raízes em plantas que recebem altos níveis de irradiância (Lei \& Lechowicz 1998, Wahl et al. 2001). Esta é uma resposta que faz sentido do ponto de vista funcional: por terem uma maior demanda evapotranspiratória, um maior investimento no crescimento do sistema radicular denso assume maior importância nas plantas sob altas irradiâncias do que nas sombreadas (Lei \& Lechowicz 1998, Poorter 1999).

De especial relevância na caracterização dos ajustes da espécie a diferentes níveis de irradiância são os parâmetros de alocação de crescimento entre estruturas aéreas (captação de luz) e subterrâneas (captação de recursos do solo). Apesar de uma menor razão entre raízes e parte aérea em plantas sombreadas ser uma resposta esperada e amplamente relatada na literatura (e.g., Luken et al. 1995, Nicotra et al. 1997, Cancian \& Cordeiro 1998, Valladares et al. 2006), esta razão não diferiu entre os dois tratamentos de luz no presente estudo (tabela 1). Experimentos em vasos conduzidos anteriormente com a mesma espécie relataram tanto resultados semelhantes (Duarte \& Dillenburg 2000), quanto contrastantes (Inoue \& Toores 1980) ao presente estudo. Uma falta de responsividade deste parâmetro a variações na irradiância também já foi relatada para outras espécies lenhosas (e.g., Callaway 1992, Luken et al. 1995, Curt et al. 2005). Duarte \& Dillenburg (2000) sugeriram que fatores como o tempo de duração do experimento e a fertilidade do substrato poderiam ajudar a explicar os resultados discrepantes dentro da mesma espécie. Valadares et al. (2006) analisaram as repostas da razão de massa entre raiz e parte aérea de quatro espécies arbóreas em níveis crescentes de irradiância e verificaram que estas respostas não eram lineares e que pouca alteração nesta razão ocorria entre níveis moderados e altos de irradiância. Da mesma forma, as diferenças nesta razão, observadas por Inoue \& Torres (1980) para a própria A. angustifolia, só se manifestaram entre os níveis altos e baixos de irradiância. No entanto, considerando-se a grande diferença nos níveis de irradiância utilizados neste trabalho e no de Duarte \& Dillenburg (2000) e partindo-se do pressuposto de que experimentos de mais longa duração, em recipientes, tenderiam a reduzir as diferenças entre tratamentos e não acentuá-las, sugere-se que o nível de fertilidade do substrato possa ser um importante fator determinante das diferenças obtidas dentro da mesma espécie.

De acordo com o esperado, a razão de área foliar foi maior nas plantas $\mathrm{S}$ do que nas plantas $\mathrm{C}$ ao final do experimento (tabela 1). Este parâmetro, juntamente com o parâmetro fisiológico taxa de assimilação líquida, determina a taxa de crescimento relativo de uma planta. Variações nesta razão em função da irradiância (menores valores a maiores irradiâncias) e do grau de tolerância ao sombreamento (maiores valores em espécies intolerantes) têm sido amplamente documentadas para diversas espécies lenhosas (e.g., Valadares et al. 2000, Walters \& Reich 1999, 2000, Lusk 2004). Com base em 16 espécies arbustivas do gênero Psychotria de uma floresta tropical, Valadares et al. (2000) demonstraram ser este parâmetro mais plástico do que a razão de massa entre raiz e parte aérea. Esta maior plasticidade pode estar relacionada ao fato dele refletir melhor e de forma mais consistente o balanço funcional entre a parte aérea e as demais partes da planta do que a razões de massa, visto que massa de um órgão nem sempre é a medida que melhor reflete sua função (Niklas 1992, Forde \& Lorenzo 2001).

Ao longo do período experimental (158 dias), as plantas de $A$. angustifolia passaram, de forma gradual, de um período de grande dependência para um período de autonomia em relação aos pinhões com os quais mantinham vínculo. Assim, não surpreende que diferenças associadas ao tamanho das plantas (massas e comprimentos) só tenham se manifestado ao final do experimento. Entretanto, alterações em padrões morfológicos (razões de massa, área e comprimento) e processos fisiológicos (acúmulo de clorofilas), caracteristicamente responsivos a diferentes níveis de irradiância, poderiam ter-se 
manifestado de forma mais antecipada ao longo do experimento. No entanto, alguns o fizeram e outros não. É possível que mecanismos de tolerância ao sombreamento pela espécie se expressem em maior grau à medida que se esgotam as reservas do pinhão. Enquanto este supre a nova planta com carboidratos, os efeitos da baixa irradiância são menores e características mais tipicamente observadas em plantas intolerantes à sombra, como um rápido alongamento caulinar, assumem um importante papel. Em persistindo as condições de baixa irradiância à medida que se esgotam as reservas do pinhão, mecanismos de tolerância à sombra fazer-se-iam-se mais importantes. Estes mecanismos parecem incluir uma alta razão de área foliar (não intermediada por uma reduzida MFA) e um alto comprimento caulinar específico.

Outras características fisiológicas associadas à tolerância ao sombreamento, como taxas fotossintéticas e respiratórias sob baixas irradiâncias e suas relações com concentrações foliares de clorofilas, constituem importantes aspectos a serem futuramente abordados, para que se possa melhor caracterizar os mecanismos de tolerância da espécie à sombra. $\mathrm{O}$ estudo de características associadas a mecanismos de conservação de recursos faz-se igualmente importante, pois a tolerância ao sombreamento nem sempre envolve um aumento na capacidade fisiológica de crescer com pouca luz, visto que grandes potenciais de crescimento podem resultar em maiores taxas respiratórias, maior reposição de tecidos, maiores danos mecânicos por herbivoria e menor estocagem (Walters \& Reich 1999). Assim, a tolerância ao sombreamento, segundo estes autores, envolve características que maximizam sobrevivência e crescimento líquido, sendo que este inclui redução de perdas por diferentes agentes, tantos bióticos quanto abióticos.

Embora o período de avaliação do presente estudo tenha sido restrito, ficou evidente que a expressão de determinados caracteres é dependente do grau de desenvolvimento das plantas. Este fato aponta para a importância já ressaltada por Lusk (2004) de se abordar as variações ontogenéticas da capacidade e dos mecanismos de tolerância das espécies a baixas irradiâncias, uma vez que, à medida que as plantas aumentam de tamanho, as ameaças à sobrevivência causadas pelo ambiente sombrio de um sub-bosque florestal podem ser alteradas. Assim, faz-se necessário um acompanhamento de mais longo prazo do desenvolvimento de plantas de $A$. angustifolia sob baixas irradiâncias. Face aos resultados contrastantes obtidos para a mesma espécie em diferentes estudos, sugere-se também que sejam investigados possíveis efeitos interativos entre irradiância e outros fatores ambientais, em particular a disponibilidade de nutrientes no solo.

\section{Agradecimentos}

As autoras agradecem ao Departamento de Solos da Faculdade de Agronomia da Universidade Federal do Rio Grande do Sul pela disponibilidade do espaço em casa de vegetação, à Coordenação de Aperfeiçoamento de Pessoal de Nível Superior (CAPES) e ao Conselho Nacional de Desenvolvimento Científico e Tecnológico (CNPq), pelas concessões de bolsas de mestrado à primeira autora e de pesquisa à segunda, e ao Programa de Pós-Graduação em Botânica pelo apoio institucional.

\section{Literatura citada}

Áquila, M.E.A. \& Ferreira, A.G. 1984. Germinação de sementes escarificadas de Araucaria angustifolia em solo. Ciência e Cultura 36: 1583-1589.

Backes, A. 2001. Determinação da idade e regeneração natural de uma população de Araucaria angustifolia (Bertol.) Kuntze em um povoamento florestal localizado no município de Caxias do Sul, RS, Brasil. Iheringia. Série Botânica 56: 115-130.

Barrett, D.R. \& Fox, J.E.D. 1994. Early growth of Santalum album in relation to shade. Australian Jounal of Botany 42: 83-93.

Boardman, N.K. 1977. Comparative photosyntheis of sun and shade plants. Annual Review of Plant Physiology 28:355-377.

Brokaw, N.V.L. 1987. Gap-phase regeneration of three pioneer tree species in a tropical forest. Journal of Ecology 75: 9-19.

Burrows, G.E., Boag, T.S. \& Stockey, R.A. 1992. A morphological investigation of the unusual cryptogeal germination strategy of bunya pine (Araucaria bidwillii) - an Australian rain forest conifer. International Journal of Plant Sciences 153: 503-512.

Cancian, M.A.E. \& Cordeiro, L. 1998. Efeito do sombreamento no crescimento inicial de Lonchocarpus muehlbergianus Hassl. Acta Botanica Brasilica 12: 367-373.

Cardillo, E. \& Bernal, C.J. 2006. Morphological response and growth of cork oak (Quercus suber L.) seedlings at different shade levels. Forest Ecology and Management 222: 296-301. 
Callaway, R.M. 1992. Morphological and physiological responses of three california oak species to shade. International Journal Plant Sciences 153: 434-441.

Curt, T., Coll, L., Prévosto, B., Balandier, P. \& Kunstler, G. 2005. Plasticity in growth, biomass allocation and root morphology in beech seedlings as induced by irradiance and herbaceous competition. Annals of Forest Science 62: 51-60.

Dale, M.P. \& Causton, D.R. 1992. Use of the chlorophyll a/ $\mathrm{b}$ ratio as a bioassay for the light environment of a plant. Functional Ecology 6: 190-196.

Duarte, L. \& Dillenburg, L.R. 2000. Ecophysiological responses of Araucaria angustifolia (Araucariaceae) seedlings to different irradiance levels. Australian Journal of Botany 48: 531-537.

Duarte, L.S., Dillenburg, L.R. \& Rosa, L.M.G. 2002. Assessing the role of light availability on the regeneration of Araucaria angustifolia (Araucariaceae). Australian Journal of Botany 50: 741-751.

Ferrer, R.S. \& Dillenburg, L.R. 2000. Efeitos da disponibilidade de luz no crescimento inicial e ecofisiologia de Scutia buxifolia Reissek (Rhamnaceae). Hoehnea 27: 147-157.

Forde, B. \& Lorenzo, H. 2001. The nutritional control of root development. Plant and Soil 232: 51-68.

Givnish, T.J. 1988. Adpatation to sun and shade: a wholeplant perspective. Australian Journal of Plant Physiology 15: 63-92.

Inoue, M.T., Galvão, F., \& Torres, D.V. 1979. Estudo ecofisiológico sobre Araucaria angustifolia (Bert.) O. Ktze.: Fotossíntese em dependência à luz no estágio juvenil. Revista Floresta 10: 5-9.

Inoue, M.T. \& Torres, D.V. 1980. Comportamento do crescimento de mudas de Araucaria angustifolia (Bert.) O. Ktze. em dependência da intensidade luminosa. Revista Floresta 11: 7-11.

Jurado, E., García, J., Flores, J. \& Estrada, E. 2006. Leguminous seedling establishment in Tamaulipan thornscrub of northeastern Mexico. Forest Ecology and Management 221: 133-139.

Knudson, L.L., Tibbilis, T.W. \& Edwards, G.E. 1977. Measurement of ozone injury by determination of leaf chlorophyll concentration. Plant Physiology 60: 606-608.

Koike, T., Kitao, M., Maruyama, Y., Mori, S. \& Lei T.T. 2001. Leaf morphology and photosynthetic adjustments among deciduous broadleaved trees within the vertical canopy profile. Tree Physiology 21: 951-958.

Lei, T.T. \& Lechowicz, M.J. 1998. Diverse responses of maple saplings to forest light regimes. Annals of Botany 82: 9-19.
Luken, J.O., Tholemeier, T.C., Kuddes, L.M. \& Kunkel, B.A. 1995. Performance, plasticity, and acclimation of the nonindigenous shrub Lonicera maackii (Caprifoliaceae) in contrasting light enviroments. Canadian Journal of Botany 73: 1953-1961.

Lusk, C. 2004. Leaf area and growth of juvenile temperate evergreens in low light: species of contrasting shade tolerance change rank during ontogeny. Functional Ecology 18: 820-828.

Morgan, D.C. \& Smith, H. 1979. A systematic relationship between phytochrome-controlled development and species habitat, for plants grown in simulated natural irradiation. Planta 145: 534-536.

Nakazono, E.M., Costa, M.C., Futatsugi, K. \& Paulilo, M.T.S. 2001. Crescimento inicial de Euterpe edulis Mart. em diferentes regimes de luz. Revista Brasileira de Botânica 24: 173-179.

Nicotra, A.B., Chazdon, R.L. \& Schlichting, C.D. 1997. Patterns of genotypic variation and phenotypic plasticity of light response in two tropical Piper (Piperaceae) species. American Journal of Botany 84: 1542-1552.

Niinemets, U., Tobias, M., Cescatti, A. \& Sparrow, A. 2006. Size-dependent variation in shoot light-harvesting efficiency in shade-intolerant conifers. International Journal of Plant Sciences 167: 19-32.

Niklas K.J. 1994. Plant allometry: the scaling of form and process. The University of Chicago Press, London.

Poorter, L. 1999. Growth responses of 15 rain-forest tree species to a light gradient: the relative importance of morphological and physiological traits. Functional Ecology 13: 396-410.

Reitz, R. \& Klein, R.M. 1966. Araucariáceas. Flora Ilustrada Catarinense. Herbário Barbosa Rodrigues, Itajaí. $62 \mathrm{p}$.

Ryser, P. \& Eek, L. 2000. Consequences of phenotypic plasticity vs. interspecific differences in leaf and root traits for acquisition of aboveground and belowground resources. American Journal of Botany 87: 402-411.

Steingraeber, D.A. 1982. Phenotypic plasticity of branching pattern in sugar maple (Acer saccharum). American Journal of Botany 69: 638-640.

Takahashi, K. 1996. Plastic response of crown architecture to crowding in understorey trees of two co-dominating conifers. Annals of Botany 77: 159-164.

Tomaselli, I. 1980. Comparação da qualidade da madeira de Araucaria angustifolia e Pinus spp produzida em reflorestamentos. In: FUPEF (ed.), IUFRO Meeting on forestry problems of the genus Araucaria, Curitiba, pp. 134-141. 
Valladares, F., Wright, S.J., Lasso, E., Kitajima, K. \& Pearcy, R.W. 2000. Plastic phenotypic response to light of 16 congeneric shrubs from a Panamanian rainforest. Ecology 81: 1925-1936.

Valladares, F., Sanchez-Gomez, D. \& Zavala, M.A. 2006. Quantitative estimation of phenotypic plasticity: bridging the gap between the evolutionary concept and its ecological applications. Journal of Ecology 94: 1103-1116.

Wahl, S., Ryser, P. \& Edwards, P.J. 2001. Phenotypic plasticity of grass root anatomy in response to light intensity and nutrient supply. Annals of Botany 88: 1071-1078.
Walters, M.B. \& Reich, P.B. 1999. Low light carbon balance and shade tolerance in the seedlings of woody plants: do winter deciduous and broad-leaved evergreen species differ? New Phytologist 143: 143-154.

Walters, M.B. \& Reich, P.B. 2000. Seed size, nitrogen supply, and growth rate affect tree seedling survival in deep shade. Ecology 81: 1887-1901.

Wintermans, J.F.G.M. \& Demois, A. 1965. Spectrophotometric characteristics of chlorophylls a and $\mathrm{b}$ ande their pheophytins in ethanol. Biochimica et Biophysica Acta 109: 448-453. 